\title{
A Single Input Model for Sequential Processing of Speech Separation
}

\author{
Motohiro Ichikawa, Naoto Sasaoka, and Isao Nakanishi \\ Graduate School of Engineering \\ Tottori University \\ Tottori, Japan \\ e-mail: nakanishi@tottori-u.ac.jp
}

\begin{abstract}
Speech separation based on auditory scene analysis (ASA) has been widely studied. A computational ASA (CASA) model, in which a mixed signal is sequentially decomposed into frequency signals, has been also proposed. Four feature types of ASA are extracted from the decomposed frequency signals, and the decomposed frequency signals are regrouped by examining the characteristics of the extracted features. Finally separated speeches are obtained. In this study, the CASA model is improved and pieced out, and the separation performance is examined via a computer simulation.
\end{abstract}

Keywords- speech separation; auditory scene analysis; single input; sequential processing; modified discrete Fourier transform

\section{INTRODUCTION}

Speech separation is actively studied worldwide. It can be applied to the hearing function of a robot, automatic generation of conference minutes, and automatic scoring of music. Speech separation involves two techniques that use multiple and unitary inputs (microphones).

As the multi-input method, blind source separation (BSS), which is a statistical method based on the independent component analysis (ICA), has gained attention. The transform (mixture) matrix from multiple inputs to measured data is estimated; then, speech separation is performed using its inverse matrix. BSS achieves superior separation performance; however, it requires an assumption that multiple sound sources are independent and that the number of microphones is greater than or equal to the number of sources.

Auditory scene analysis (ASA) is proposed as the unitary input method [1]. Human beings can hear specific speeches in an environment where people speak simultaneously. This ability is well known as the cocktail party effect. The ASA psychologically explains the auditory mechanism of human beings. A mixed speech can be separated by extracting four features: common onset/offset, harmonic structure, common changes, and gradual changes. Then, the extracted features are grouped.

Computational ASA (CASA) processes ASA in a computational algorithm [2], which is based on the timefrequency analysis (spectrogram) obtained via block processing. In addition, the separation performance of mixed speeches and the reproducibility of original speeches will be improved by adopting a leaning function [3]-[11], in which all features are extracted in advance for separation. The unitary input method can eliminate the condition that the number of microphones has to be greater than or equal to that of the sources.

This study aims to realize CASA in sequential processing, which is more suitable for real-time processing than block processing. In contrast, the separation performance may be degraded as the available features in a sampling period are restricted compared with block-processing models. This study also aims to investigate how the four features of ASA are implemented in the sequential processing and to clarify what the sequential processing of CASA can and cannot accomplish.

A basic model for the sequential processing of ASA has been proposed previously [12]. However, only the harmonic structure feature has been implemented and evaluated. In this study, the detection of the harmonic structure feature is improved and the remaining three features are implemented.

\section{Sequential Processing Model of ASA}

ASA has been proposed to provide a framework for clarifying the auditory function of human beings [1]. In ASA, four physical features in a mixed signal, namely "common onset/offset," "harmonic structure," "common change," and "gradual change" play prominent roles.

We have proposed to realize sequentially the unitary input model of ASA using a modified discrete Fourier transform (MDFT) pair [13], which is illustrated in Fig. 1. A mixed sound $x_{i}$ is sequentially decomposed into frequency signals using MDFT. From the frequency signals, the four features of ASA are extracted by the detectors. In the grouping controller, the group to which each frequency signal belongs to is determined using the extracted features. The grouped signals are added in the modified inverse DFT (MIDFT) and a separated sound is generated. Please refer to Ref. [12] in details.

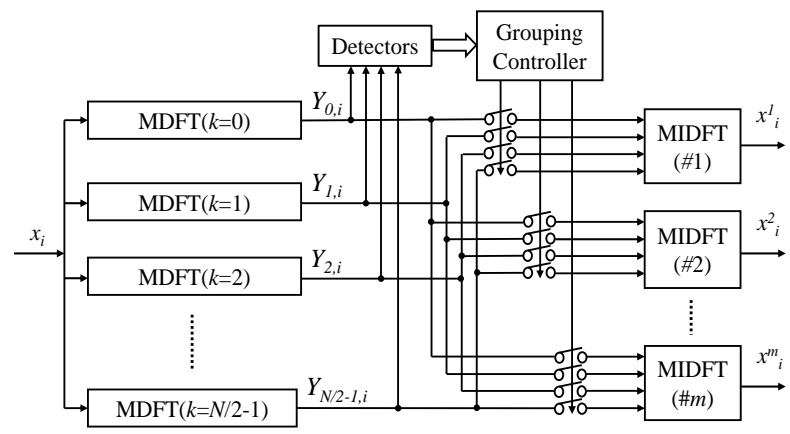

Figure 1. Sequential processing of ASA using a MDFT pair. 


\section{A. Detectors}

Before the detection of four features, the frequency signals decomposed from an input signal are averaged using a moving window with 100 samples. Then, their envelopes are detected using the signal level detectors proposed in Ref. [14] to examine the global behavior of each signal.

\section{1) Detection of common onset/offset}

For detecting the common onset/offset features in frequency signals, the onset/offset points in each signal are determined. If the amplitude of a signal at a given time is smaller or greater than a threshold, a label with the value " 0 " or " 1 ," respectively, is applied. The time when the value " 0 " changes to " 1 " is regarded as an onset point. Inversely, the time when the value " 1 " changes to " 0 " is regarded as an offset point. The threshold value must be adjusted according to the amplitude of an input.

2) Detection of common changes

The variation of a frequency signal is represented by an increase or decrease of the signal. The amplitude of a frequency signal at a given time is subtracted from that in previous 100 sampling periods. If the result is positively larger than a threshold, " +1 ” is assigned at the frequency, whereas " -1 " is assigned in the negative case. If the result is smaller than the threshold, " 0 " is assigned. If the frequency signals have the same value at the same time, they are regarded as being commonly changing.

3) Detection of harmonic structure

The harmonic structure is the backbone of processing in the proposed model and the detection accuracy of this structure greatly influences the speech separation performance. In Ref. [12], harmonic structures were extracted and the fundamental frequencies were determined using the harmonics; however, this caused misdetection wherein the grouped harmonics included unnecessary frequency signals. In this study, an improved detection method of harmonic structures is introduced to detect the fundamental frequencies.

The extraction method of spectral peaks is identical to that used in the conventional method, which is not novel and its concept is described in Ref. [15] for example. However, the detected peaks under the decided frequency are regarded as the fundamental frequencies in the proposed method, whereas all detected spectral peaks are candidates for the fundamental frequencies in the conventional method. Harmonic frequencies are estimated based on the phenomenon that the frequencies of harmonics are integral multiples of the fundamental frequency. However, this phenomenon is not always true. The frequency values of harmonics slightly vary in actual voicing samples.

Let us explain the issue using Fig. 2 where the spectral peaks are detected at frequency $k=16,30$, and 45 and then the fundamental frequency is regarded as 16 . However, if the harmonics searching using the fundamental frequency of $k=16$ never detect its harmonics of $k=30$ and 45 . The fundamental frequency may be changed from its true value because of its variation.

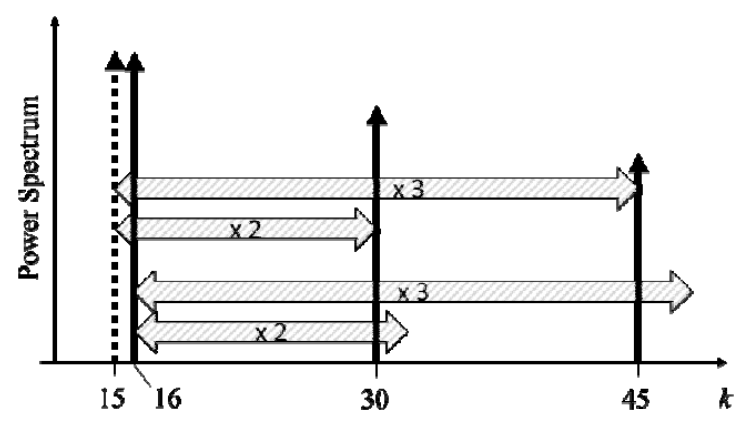

Figure 2. An example of variation of harmonics.

Another issue is that the spectral peaks of all harmonics are never simultaneously detected. Harmonics in a real speech does not always vary simultaneously as they have different amplitudes and phases. Figure 3 shows an instance of the time variation of the detected spectral peaks at frequencies $k, 2 k$, and $3 k$, which correspond to the harmonics in a real speech. Even in harmonics, their spectral peaks can never be extracted synchronously. This phenomenon poses a problem in sequential processing as it causes the misdetection of harmonics. For addressing this problem, \pm 1 frequencies of the integral-multiple frequencies of the fundamental frequency are also regarded as harmonics. In contract, the fundamental frequency also varies slightly; therefore, the fundamental frequency and its \pm 1 frequencies are used for estimating harmonics. In other words, the above integral multiplication is always achieved at three frequencies (the fundamental frequency and its plus and minus 1 ones. From the three candidates obtained, the frequency with the largest number of harmonics is determined as the true fundamental frequency and its integral-multiple frequencies are detected as harmonics.

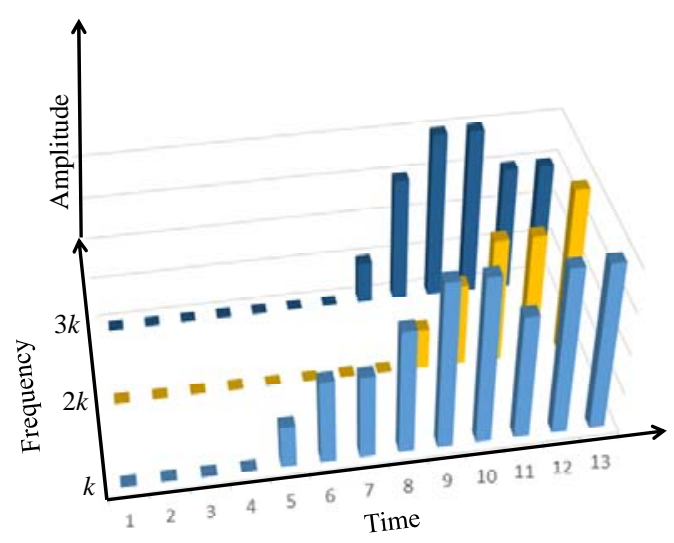

Figure 3. Time variation of the detected spectral peaks.

In this study, a moving-window method is introduced for mitigating the misdetection. Let us explain the proposed method using Fig. 4, where " 1 " indicates the existence of spectral peaks and $2 f$ denotes the window size. 


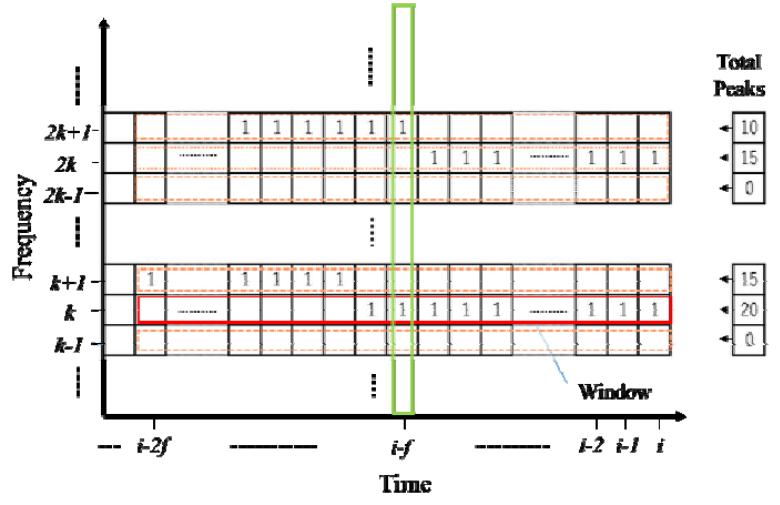

Figure 4. Time variation of the detected spectral peaks.

Assuming the current time is $i$, the detection of the harmonic structure is always examined at $i-f$. In Fig. 4 , it is assumed that there are two spectral peaks at $k$ and $2 k+1$ at $i-f$ and the lower peak corresponds to the fundamental frequency. In addition, the number of spectral peaks in a window is assumed to be 20 at $k$ and those at $k+1$ and $k-1$ are 15 and 0 , respectively. In this case, the maximum value is 20 at $k$. Similar counting is achieved at harmonics and their \pm 1 frequencies. In Fig. $4,2 k$ is assumed as the second harmonic. Assuming that the number of spectral peaks in a window at $2 k, 2 k+1$, and $2 k-1$ are 15,10 and 0 , respectively, the maximum value is 15 at $2 k$. By adding both the maximum values, 20 and 15 , the total value of 35 is obtained for $k$.

For dealing with the variation in the fundamental frequency, \pm 1 frequencies of the fundamental frequency $k$ are also investigated using the above procedure. In Fig. 4, in the case of $k+1$, the number of spectral peaks in a window is assumed to be 15 and those at the second harmonic to be $2(k+1)$ and its \pm 1 frequencies, $2(k+1)+1$ and $2(k+1)-1$, are 0 ; 0 ; and 10 , respectively. The total number of spectral peaks is 25 at $k+1$. In the case of $k-1$, the total value is assumed to be 0 at $2(k-1)$ and in the case $k+1$, it is 0 . Comparing the total values, $k$ with the maximum total value of 35 is regarded as the true fundamental frequency. Harmonics are sought using this fundamental frequency.

The window size is considered as a grace period for determining the fundamental frequency. In contrast, such a grace period causes a delay in processing and should be minimized; in the proposed method, $f$ sampling periods are grace periods.

4) Gradual change

In ASA, the gradual change comprises two characteristics, namely, "similarity" and "continuity" as shown in Fig. 5. Similarity is defined as the connectedness of sound for a short time (e.g., in a phoneme), and the continuity provides a criterion for the connectedness of sound for a long time (e.g., in successive phonemes).

a) Detection of similarity

Similarity is based on the phenomenon that the fundamental frequency does not change considerably. The

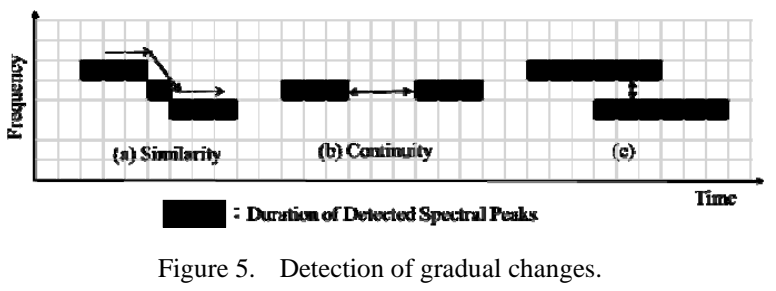

fundamental frequency can be obtained via the detection of harmonic structures. If the detected fundamental frequencies are successive and have the same value, they are regarded identical. However, even if the successive fundamental frequencies are different and have small variation width, they are regarded as identical. When the fundamental frequency is $k$ at time $i$ and that at the previous sampling period $i-1$ is within $k \pm 1$, both fundamental frequencies are regarded as connected.

\section{b) Detection of continuity}

The continuity is also based on the phenomenon that the characteristic of a sound never changes considerably. The fundamental frequency never changes suddenly in a few phonemes. However, if the phonemes include silent zones, the method for detecting the similarity cannot be used to detect the continuity and another method for detecting continuity is required. The key point is to utilize the information of fundamental frequencies that were included in previously grouped harmonics.

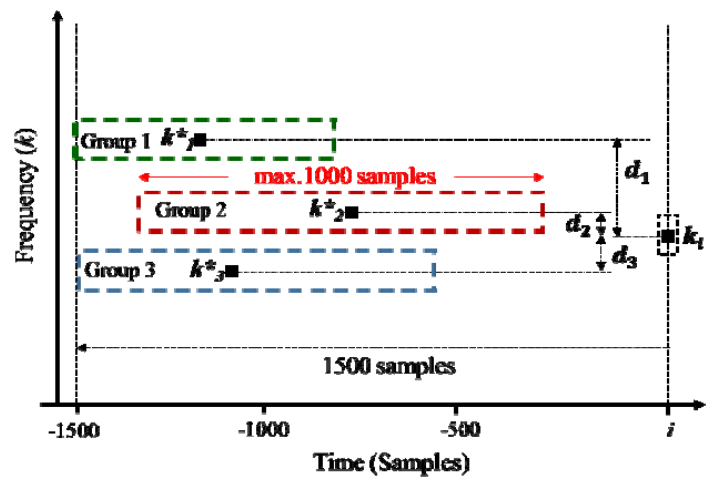

Figure 6. Detection of Continuity.

Let us explain the procedure using Fig. 6. When a new harmonic structure with a fundamental frequency $k_{i}$ is detected at $i$ and there is no fundamental frequency before just one sampling period, it is examined whether harmonic groups are present during the past 1500 sample periods from the current time. The duration corresponds to the number of samples in a phoneme when the sampling frequency is $8 \mathrm{kHz}$. If there are harmonic groups, the fundamental frequencies during the maximum 1000 sampling periods are averaged in each harmonic group. If the difference $d_{n}$ between the fundamental frequency $k_{i}$ of the detected harmonic structure and the averaged frequency $k_{n}^{*}$ is within \pm 4 and is the smallest, the harmonic structure with the smallest difference is regarded as connected to the detected harmonic structure. 
Moreover, another issue has to be resolved. Two fundamental frequency peaks are simultaneously detected at different frequencies; however, these are originally identical as indicated in Fig. 5(c). This phenomenon is caused by a variation in the fundamental frequency and the characteristic of the signal level detector, which has a large time constant for a decreasing signal [14]. For instance, it can be assumed that two phonemes are successive; then, the former's fundamental frequency is changed in the latter phoneme. Even after the former phoneme ends, the frequency signal as an output of the signal level detector is continued because it is filtered using a large time constant. Therefore, an originally identical fundamental frequency is detected at two frequencies.

To solve this problem, the onset/offset feature is utilized. Let us explain that using Fig. 7. Actual voiced speeches do not comprise only line spectra; therefore, they comprise main lobes and side lobes. The detected spectral peaks correspond to the main lobes. Preferring to the onset/offset feature of the side lobes, continuity is detected even if an originally identical fundamental frequency is detected at two frequencies as described below.

When a spectral peak of the fundamental frequency ends at (a) in Fig. 7, the similarity and the continuity at this point are investigated first. Even if they are not detected, the presence or absence of another spectral peak is examined within its \pm 3 frequencies. If there is another spectral peak at (b), the onset/offset features of the fundamental frequency and those of the frequency of another peak are compared. If the onset/offset features are common, those spectral peaks are regarded to correspond to an identical fundamental frequency, i.e., the spectral peaks are continued.

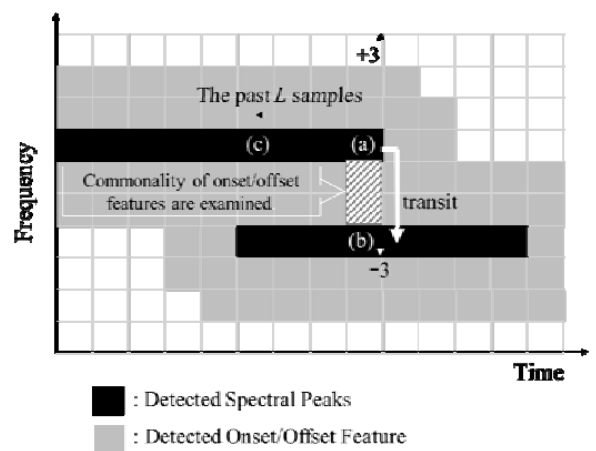

Figure 7. Gradual change detection using the common onset/offset feature.

In contrast, if the detected spectral peaks at different frequencies do not correspond to the identical fundamental frequency but to different fundamental frequencies, the above processing causes the misdetection of continuity. To address this problem, even if the continuity is detected after the above processing, the amplitudes of two frequency signals are compared with each other. However, this comparison is performed not at the end-point of a detected spectral peak (a) but at a point beyond the past $L$ sampled point at (c) from the end-point. If the difference between the amplitude levels is less than a quarter of a major one, the two detected spectral peaks at different frequencies are concluded to correspond to an identical fundamental frequency.

\section{B. Grouping Controller}

In the grouping controller, harmonic structures are first extracted from spectral peaks based on the method described in Sect. II-A3 and frequency elements (harmonics) in each harmonic structure are grouped. Next, the common change is examined in each group as described in Sect. II-A2. Concretely, the number of “ +1 "s, “ -1 "s, or " 0 "s of the spectral peaks is investigated in each group. If the number of " +1 "s is larger than half of the number of all peaks, the group is regarded as increasing and all spectral peaks are grouped even if some spectral peaks have " -1 ” or " 0 .” This processing for grouping is performed up to \pm 3 frequencies of each spectral peak. However, if the number of " +1 "s of the frequency element is less than half of the number of all spectral peaks, this processing is aborted. The above processing is also achieved similarly for " -1 " and " 0 ." If the number of spectral peaks with the same value is less than half of the number of all peaks, the detected onset/offset is examined described in Sect. II-A1. If the harmonics are labeled as the onset, they are also grouped. This processing is performed at \pm 3 frequencies of each harmonic. Finally, the similarity and continuity are investigated as described in Sect. II-A4 and the time-connectedness of grouped harmonics is guaranteed.

\section{EVALUATION IN EXPERIMENTS}

Figures 8 (a), (b), and (c) show the waveforms of a mixed signal, male speech, and female speech used for performance evaluation, respectively. The number of samples for MDFT was $N=768$; therefore, the maximum frequency is $N / 2-1=383$. The upper limit for estimating a fundamental frequency was $k=40$. The threshold for extracting spectral peaks was the sum of 100 and the twofold mean of the input spectrum. $L$ for comparing the amplitude levels was 100 . The moving-window size $2 f$ for detecting the fundamental frequency was 101; therefore, a processing delay of 50 sampled periods, i.e., $6.25 \mathrm{~ms}$ is necessary. The thresholds for detecting the common change and the common onset/offset are set to

$$
2 \mathrm{X}+100+(400 / k) \text { for } 1 \leq k<90 \text {, }
$$$$
2 \mathrm{X}+50+(50 /(k-89)) \text { for } 90 \leq k \text {, }
$$

where $\mathrm{X}$ is the mean value of the amplitude spectrum.

The results are shown in Figs. 8 (d) and (e). The proposed method cannot determine which separated signal corresponds to the original speech. The separated signals (d) and (e) seem to be the original signals (b) and (c), respectively. Herein, the performance was evaluated additively using five mixed signals and the equivalent results were obtained. It is roughly confirmed that speech separation based on ASA can be achieved sequentially using the proposed model. Degradation naturally occurs in the separated signals as the information that can be used in the 
proposed sequential processing is restricted in contrast with that of the conventional block-processing methods. As the performance of detectors for four features is improved, the separation performance will be improved. However, when two frequency signals have the same frequency and the same variation (phase), it is difficult to separate them using the proposed model. This is an apparent limitation of the proposed model.

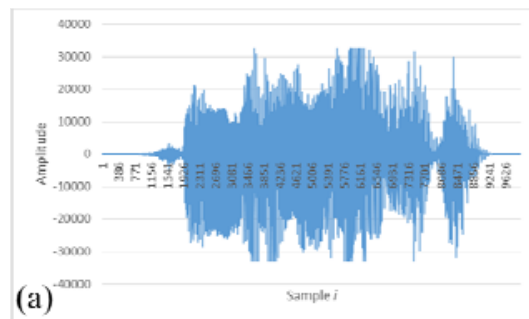

IV. CONCLUSIONS

A basic model for the sequential processing of ASA was proposed herein. In the conventional system, only the detection of the harmonic structure feature was implemented. In this study, the detection performance of the harmonic structure feature was improved and the detectors for the remaining features were implemented. Speech separation performance was evaluated using mixed speeches. The results confirmed that the sequential processing of ASA using the proposed model was feasible; however, speech separation could not be completely achieved using the proposed model.

A future study must involve the verification of the separation performance using various mixed signals, the objective evaluation based on the similarity, and the subjective evaluation of the separation performance using experimental subjects.

\section{REFERENCES}

[1] A. S. Bregman, "Auditory Scene Analysis: Hearing in Complex Environments," Chapter 2 in S. McAdams and E. Bigand (Eds.), Thinking in Sound, Oxford Univ. Press, 1992.

[2] D. Wang and G. J. Brown, "Computational Auditory Scene Analysis,” IEEE Inc., 2006.

[3] Y. Shao, S. Srinivasan, Z. Jin, and D. Wang, “A Computational Auditory Scene Analysis System for Speech Segregation and Robust Speech Recognition,” Computer Speech and Language, vol. 24, pp. 77-93, 2010.

[4] P. Li, Y. Guan , S. Wang , B. Xu, and W. Liu, "Monaural Speech Separation Based on MAXVQ and CASA for Robust Speech Recognition,” Computer Speech and Language, vol. 24, pp. 30-44, 2010 .

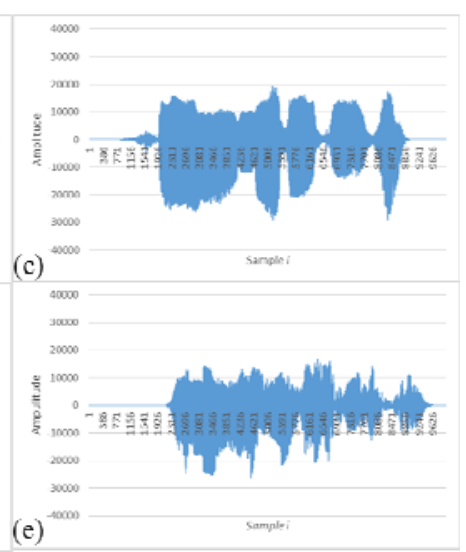

(e)

aveforms.

[5] M. Cooke, J. R. Hershey , and S. J. Rennie, "Monaural Speech Separation and Recognition Challenge", Computer Speech and Language, vol. 24, pp. 1-15, 2010.

[6] C. Hsu, and J. R. Jang, "On the Improvement of Singing Voice Separation for Monaural Recordings Using the MIR-1K Dataset," IEEE Trans on Audio, Speech, and Language Processing, vol. 18, No. 2, pp. 310-319, 2010.

[7] G. Hu and D. Wang, “A Tandem Algorithm for Pitch Estimation and Voiced Speech Segregation,” IEEE Trans on Audio, Speech, and Language Processing, vol. 18, No. 8, pp. 2067-2079, 2010.

[8] Z. Jin and D. Wang, "Reverberant Speech Segregation Based on Multipitch Tracking and Classification," IEEE Trans on Audio, Speech, and Language Processing, vol. 19, No. 8, pp. 2328-2337, 2011.

[9] A. Rabiee, S. Setayeshi, and S. Lee, “A Harmonic-Based Biologically Inspired Approach to Monaural Speech Separation,” IEEE Signal Processing Letters, vol. 19, No. 9, pp. 559-562, 2012.

[10] A. Mahmoodzadeh, H. R. Abutalebi, H. Soltanian-Zadeh, and H. Sheikhzadeh, "Single Channel Speech Separation in Modulation Frequency Domain based on a Novel Pitch Range Estimation Method,” EURASIP Journal on Advances in Signal Processing, 2012.

[11] W. Yu, L. Jiajun, C. Ning and Y. Wenhao, "Improved Monaural Speech Segregation Based on Computational Auditory Scene Analysis," EURASIP Journal on Audio, Speech, and Music Processing, 2013.

[12] I. Nakanishi, J. Hanada, “A Sequential Processing Model for Speech Separation Based on Auditory Scene Analysis,” Proc. of 2015 IEEE International Symposium on Intelligent Signal Processing and Communication Systems (ISPACS2015), pp. 124-128, Nov. 2015.

[13] S. Yoneda, I. Nakanishi, I. Sasaki, and A. Ogihara, "Switchedcapacitor DFT and IDFT circuit,” Int. J. Electronics, vol. 67, no. 6, pp. 839-851, Dec. 1989.

[14] Y. Minato and I. Nakanishi, "Noise Reduction System Using Signal and Noise Level Detectors in Frequency Domain,” Proc. of 2008 IEEE International Symposium on Intelligent Signal Processing and Communication Systems (ISPACS2008), pp. 180-183, Mar. 2009.

[15] X. Serra and J. O. Smith, "Spectral Modeling Synthesis," Proc. of International Computer and Music Conference, pp. 281-284, Nov. 1989. 